\title{
Hybrid Data Transmission Algorithms for Energy Balancing in Wireless Sensor Networks
}

\author{
Kavi Kumar Khedo \\ Department of Computer Science and Engineering \\ University of Mauritius \\ Reduit, Mauritius
}

\author{
Bhama Imrith \\ Department of Computer Science and Engineering \\ University of Mauritius \\ Reduit, Mauritius
}

\begin{abstract}
One of the main research problems in the area of wireless sensor network has been to prolong life-time of network by using energy efficient algorithms. Sensor nodes in WSNs has different energy consumption rate due to the uneven complexity of the sensing tasks and varying distance from sink node. Consequently this leads to fast energy disparity among the sensor nodes and unbalanced energy state in the network, which in turn result in short lifetime of the network. Therefore balancing energy consumption, in order to prolong network lifetime has become an important aim for WSNs.

In this paper two algorithms are being proposed to better balance the energy dissipation in heterogeneous cluster-based WSNs. The first algorithm is called unequal width hybrid communication and the second is the equal width hybrid routing communication. Both algorithms use a hybrid data transmission mode which consists of a probability based energy balancing scheme that divides the network into $\mathrm{K}$ rings of unequal and equal widths respectively and find a set of probabilities $\mathrm{Pi}$ for each ring to balance the energy consumption. Simulation results have shown the validity of the algorithms which not only minimize the total energy consumption, but also balance the average per-node energy consumption across all nodes and hence prolong the sensor network lifetime.
\end{abstract}

\section{Categories and Subject Descriptors}

C.2.2 [Computer Communication Networks]: Network Architecture and Design - network communications, network topology, wireless communication.

\section{General Terms}

Algorithms, Design, Experimentation, Performance.

\section{Keywords}

Sensor Networks, Energy Balancing, Hybrid Communication Algorithm, Clustering, Multi Hop Communication.

\section{INTRODUCTION}

Recent advances in Micro-Electro Mechanical Systems (MEMS) have led to the development of small sensor nodes that are composed of embedded devices like communication boards and sensor boards [1]. However smart sensors have several limitations - limited power, prone to failure and limited computing capabilities. Because replenishment of the battery of sensor nodes is very difficult, lifetime of WSNs is highly constrained. The difficulty of battery replenishment is caused by the hostile environments in which the sensor nodes operate. Therefore the study of energy efficient scheme to prolong network lifetime in WSN has been a very active area of research $[1,2]$. The lifetime of wireless sensor network is crucial, since autonomous operation must be guaranteed over an extended period.

Sensors are severely constrained by the energy of their batteries, while the network lifetime is expected to be as long as possible. So saving energy to maximize the network lifetime is one of the critical problems in WSNs. In order to prolong the network life, two different problems need to be solved. One is the inefficient energy consumption and the other is the unbalanced energy consumption. The latter is an inherent problem in wireless sensor networks and it is largely orthogonal to the general energy efficiency problem $[3,4]$.

For example, we assume that every sensor node in the network has an equal probability of generating data packets that have to be forwarded to the base station via multi-hop routing using other sensor nodes as relays. Apparently, the burden on the nodes close to the base station is considerably higher than on the nodes that are far away. Inevitably, the nodes closer to the sink will experience higher traffic and higher energy consumption rate. These nodes will be the first ones which run out of power [4, 5] and thus compromising the proper functioning of the overall network. Without appropriate measures, they will die quickly, rendering the network useless. In this paper, we propose and discuss strategies to ensure maximum lifetime of the network by balancing the energy load as equally as possible.

\section{RELATED WORKS}

Energy is the scarcest resource in the wireless sensor node and the energy constraint is unlikely to be solved due to slow progress in battery capacity. The sensor nodes are battery-driven and are often deployed in a hazardous and inaccessible environment; therefore battery replacement is not a feasible solution and human intervention is also undesirable since some networks consist of hundreds to thousands of nodes, which imply high operational cost [6]. Such large physically distributed networks increase the difficulty of changing batteries and makes recharging almost impossible during operations. This problem has forced the node, network and system developers to make changes in the basic WSN architecture as well as the researchers to develop new 
algorithms to minimize the energy consumption especially of the nodes, thereby maximizing the network lifetime [7, 8].

Energy balancing is one of the challenging topics in wireless communication that has been proposed for WSNs [3]. The idea behind energy balancing is to spread the energy consuming tasks across the nodes so as to obtain a more balanced energy consumption and uniform energy distribution (i.e. the "average" energy dissipation per sensor is at each time the same and no node dies early due to excessive usage). Keeping up the uniform energy consumption across sensor nodes has been proposed as an effective approach to prolong the lifetime of WSNs [3, 4].

The unbalanced energy consumption phenomenon, which is an inherent problem in WSN, arises because sensor nodes usually collaborate with each other via multi-hop links. The multi-hop organization presents many advantages, from the increase of the network capacity, ability to perform data fusion and more efficient energy utilization. However, under many scenarios, multi-hop sensor networks utilize energy in an unbalanced manner. To illustrate this phenomenon, consider the simple, unidirectional example in Figure 1.

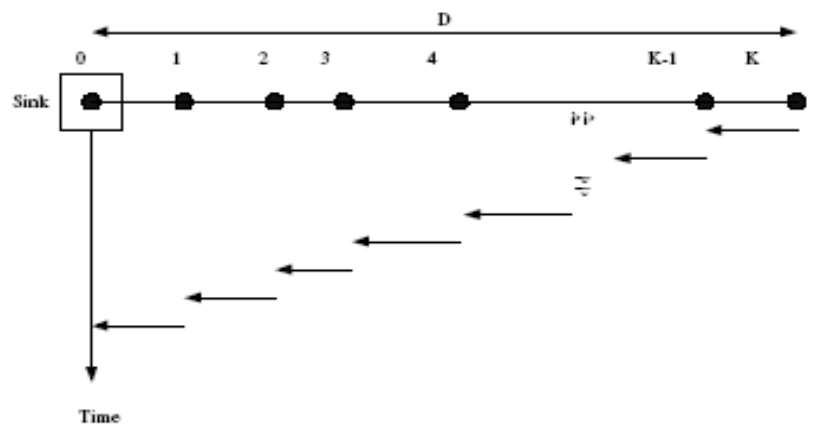

Figure 1: $(K+1)$-node line network connected [4]

It is assumed that all nodes communicate only with their neighbors and all the nodes are sending their observations back to the sink. It is also assumed that the nodes are equidistant, and thus the dissipated energy being roughly the same for each node. Normally, if all nodes have the same initial energy level upon deployment, the node closer to the sink will drain earlier since it has heavier forwarding burden. Moreover, the further nodes which may still have plentiful energy supplies cannot find the routes to the sink. The energy unbalancing problem will deteriorate with the increase of the network depth [4].

Furthermore this unbalanced energy consumption is a common problem in clustering wireless sensor networks and it is largely orthogonal to the general energy efficiency problem [4]. In a clustering network, nodes are partitioned into a number of small groups called clusters. Each cluster has a coordinator, referred to as the cluster-head, and a number of member nodes. Member nodes route to their cluster-head to report collected data as shown in figure 2 [9]. The cluster-heads transmit data over long distances to the base station by one-hop or multi-hop. In a cluster with multi-hop mode, the sensor nodes closest to the cluster-head have the highest energy consumption due to packet relaying, so they tend to deplete their energy budget faster than other sensors.

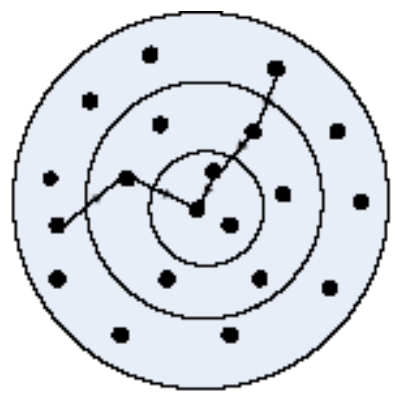

Figure 2: Routing to cluster-head

Another problem in cluster-based networks is that some clusters are high activity regions where data transmission, communication and monitoring are frequent. Without appropriate measures, the nodes will be depleted of their energy quickly, rendering the network useless. Therefore, to overcome the problems mentioned above, researchers have proposed different energy balancing algorithms $[10,11,12,13]$ with the following benefits:

- To ensure that every sensor node has the same rate of energy dissipation (or as close as possible) such that the network remains functional for the maximum possible time.

- To balance the energy load and maintain connectivity as equally as possible.

- To ensure uniform energy drainage, i.e. all the nodes deplete energy at the same rate.

\subsection{Unequal width multi-hop communication}

Sun et al. proposed the unequal width multi-hop communication mode [9] which is an energy balancing technique that allows the sensors to adjust their transmission distances accordingly so as to send data to the cluster-head $(\mathrm{CH})$ using multi-hop communication, therefore leading to uniform energy consumption rate across the sensor nodes in the cluster. Figure 3 shows a cluster divided into $\mathrm{K}$ concentric rings, the radii of $\mathrm{K}$ concentric rings centered at the cluster-head are $0<=\mathrm{r}_{0}<\mathrm{r}_{1}<\mathrm{r}_{2}<\ldots<\mathrm{r}_{\mathrm{K}}=\mathrm{R}$ (depending on the value $\alpha$ ), and the width of the $i^{\text {th }}$ ring is $\Delta_{\mathrm{i}}$, which equals $r_{i}-r_{i-1}$, where $i=1,2, \ldots, K$.

The authors have assumed a heterogeneous sensor network with uniform distribution and an energy model $E=a\left(d^{\alpha}+c\right)$ where $\alpha$ is the energy attenuation power parameter related to a specific field $(2<=\alpha<=6), d$ is the distance between the sender and receiver and $\mathrm{c}$ is a technology dependent positive constant.

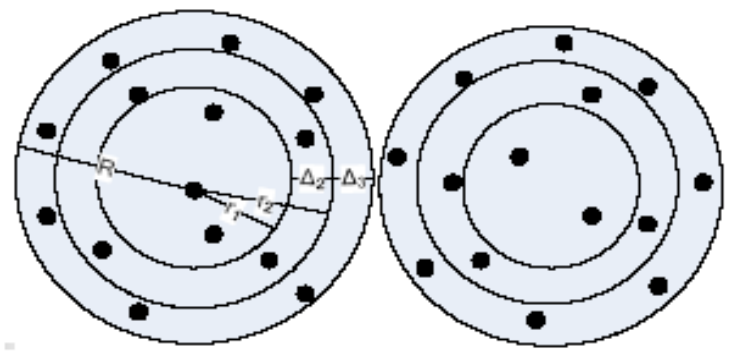

Figure 3: A clustering sensor network [9] 
The proposed algorithm achieves the uniform energy consumption of nodes by taking different transmission radii according to the distance of sensor nodes to the cluster-head.

\subsection{Equal width hybrid communication}

Sun et al. proposed a second algorithm which balances energy consumption across all the rings by equal widths design. The equal width hybrid communication mode [9] allows the sensor nodes to communicate with their cluster-heads with constant transmission radii and mixed communication modes instead of only multi-hop mode or single-hop mode. The authors have assumed that the sensors in different rings will have different probabilities to communicate with the $\mathrm{CH}$ so as to ensure uniform energy consumption. The hybrid communication mode has been proposed as follows: the sensor nodes in the $\mathrm{i}^{\text {th }}$ ring are allowed to send their packets to the cluster-head by one-hop with probability $\mathrm{p}_{\mathrm{i}}$, which means a packet in the $\mathrm{i}^{\text {th }}$ ring will be sent to the $(\mathrm{i}-1)^{\text {th }}$ ring with probability $1-p_{i}$ and to the cluster-head directly with probability $\mathrm{p}_{\mathrm{i}}$.

\subsection{Energy Balanced Transmission (EBT)}

EBT [14] is a transmission scheme that balances energy consumption among nodes by considering transmission distance (between the nodes and the base station) and data density. The two mentioned factors affect the energy consumption rate of the sensor nodes as follows:

- If the data density is high or the node is far away from the base station, this means that it may need more power than the other nodes to transmit data. Therefore more percent data can be sent to the next hop, which may reduce the total energy consumed by that node.

- However, if the data density is low or the node is close to the base station, more data can be sent directly to the base station.

The authors have considered a division of the network into slices, composed of sensors at nearly equal distance to the sink and able to send the data to sensors belonging to the next slice (toward the sink) or directly to the sink. EBT then adjusts the ratio of the data transmitted to the next hop and to the base station so as to achieve a constant energy consumption rate.

\subsection{Routing strategy}

In [15], the author proposed and analyzed four energy balancing strategies that are used to balance the energy consumption of the nodes, where one of them is the routing strategy. The latter is similar to the equal width hybrid communication except that here it has been assumed that a node will transmit the locally generated traffic to the next node with probability $a_{i}$ and directly to the sink with probability $b_{i}=1-a_{i}$ while incoming traffic will always be forwarded to the next node. The goal is to find $a_{i}$ so as to achieve energy balancing. Furthermore the algorithm is based on the 'Rayleigh fading link model' which is represented by $E=d^{\alpha} \Theta \sigma^{2} /-\ln P_{L}$ rather on the 'disk model'.

\section{PROPOSED ENERGY BALANCING ALGORITHMS}

In this section we proposed two algorithms that balance the energy consumption of the nodes to increase the lifetime of the network substantially. The algorithms have been designed in order to satisfy the following broad requirements:

- The algorithms should ensure evenness in the energy consumed by the sensor nodes in a network. Therefore the new algorithm must balance the energy consumption rates efficiently across the sensor nodes. To achieve this, the energy balancedness property must be satisfied and it is as follows: $\mathrm{E}_{1}=\mathrm{E}_{2}=\ldots=\mathrm{E}_{\mathrm{N}}$ meaning that the average per sensor energy dissipation is the same.

- The algorithms should improve the lifetime of the network considerably through uniform energy dissipation.

- The algorithms should optimize the energy consumption so as to save energy during the routing process as well as prolong the network lifetime.

- The algorithms should distribute the energy load evenly across all the sensor nodes, especially the critical nodes (i.e. the nodes closest to the cluster head which are responsible for relaying the data to the base station from the other nodes) and thereby avoid the 'hot spot' problem in WSN.

- The algorithms should employ the hybrid transmission technique which consists of both direct and multi-hop transmissions.

- The algorithms should be simple so as to occupy the least amount of memory space and be computationally fast.

\subsection{Proposal 1: Unequal Width Hybrid Communication Mode}

The unequal width hybrid communication mode (referred as unequal width) is a combination of the hybrid communication algorithm and the unequal width design. The hybrid communication mode will consist of a probability based energy balancing scheme that will divide a circular network into $\mathrm{K}$ rings of unequal widths and will find a set of probabilities $\mathrm{P}_{\mathrm{i}}$ for each ring to balance the energy consumption. The node in ring $i$ will then use the calculated probability $P_{i}$ to determine the number of data packets to be forwarded to the next ring $i-1$ towards the $\mathrm{CH}$ and to the $\mathrm{CH}$ directly respectively. The unequal width algorithm will calculate the optimized transmission range for each ring, which will be used in the process of data routing, in order to save energy and thereby maximize the network lifetime.

\subsubsection{The unequal width design}

The unequal width design has been included in the scheme to make the average energy consumed per node minimal. Therefore it must find the optimal transmission ranges of the rings in order to achieve the minimum balanced energy consumption and extend the network lifetime longer. To achieve this, the following formula has been considered:

$$
E=\sum_{i=1}^{k} a\left(h_{i}^{\alpha}+c\right), \sum_{i=1}^{k} h_{i}=D
$$

In order to minimize $\mathrm{E}$, all hop distances $\mathrm{h}_{\mathrm{i}}$ are made equal to $\mathrm{D} / \mathrm{k}$.

$$
E=a c^{*} k+a h_{i}^{\alpha}+a h_{2}^{\alpha}+\ldots+a h_{k}^{\alpha}=a c k+a k\left(\frac{D}{k}\right)^{\alpha}=a c k+a D^{\alpha} k^{1-\alpha}
$$

Let the derivative of $\mathrm{E}$ with regard to $\mathrm{k}$ be: 


$$
E^{\prime}=a c-\frac{(\alpha-1) a D^{\alpha}}{k^{\alpha}}
$$

When $E^{\prime}$ is made equal to 0 , the following is obtained:

$$
\frac{D}{k}=\mathrm{r}_{\mathrm{opt}}=\sqrt[\alpha]{\frac{c}{\alpha-1}}
$$

Where $\mathrm{D} / \mathrm{k}=$ optimal transmission range $\left(\mathrm{r}_{\mathrm{opt}}\right)$ for the multi-hop mode.

It is decided that only the width of the $1^{\text {st }}$ ring will be equal to the optimal transmission range obtained above, i.e. $r_{1}$

$=\alpha \sqrt{\frac{c}{(\alpha-1)}}$. The optimal transmission ranges of the ring $i(1<=$ $i<=\mathrm{K}$ ) will be calculated as follows, assuming that the number of rings is $\mathrm{K}$ :

If $\alpha=2$, let $r_{1}=r_{\text {opt }}$; the sequence $r_{1}, r_{2}, r_{3}, \ldots, r_{k}$ decreases (meaning that the ring widths must decrease with the increasing of i).

Else if $\alpha=3$, then $r_{1}=r_{\text {opt }}$; the sequence $r_{1}, r_{2}, r_{3}, \ldots, r_{k}$ must wave.

Else if $\alpha=4$, then $\mathrm{r}_{1}=\mathrm{r}_{\mathrm{opt}}$; then the sequence $\mathrm{r}_{1}, \mathrm{r}_{2}, \mathrm{r}_{3}, \ldots, \mathrm{r}_{\mathrm{k}}$ must increase.

\subsubsection{The hybrid communication algorithm}

To remedy the energy imbalance problem among the rings, the hybrid scheme based on probability is introduced. It calculates the total number of packets present in ring $i$ as follows:

$N_{i}=N_{i}^{L}+N_{i}^{i n c}$

Where $N_{i}^{L}=m_{i}=\lambda \pi\left(R_{i}^{2}-R_{i-1}^{2}\right)$,

$N_{i}^{i n c}=\left(1-p_{i+1}\right) N_{i+1}$

$N_{i}=N_{i}^{L}+\left(1-p_{i+1}\right) N_{i+1}$

(for $i=1,2 \ldots \mathrm{K}-1$ )

Where $p_{i+1}$ is the probability that a packet in the $(i+1)^{\text {th }}$ ring will be sent directly to the cluster-head.

Then it then determines the total energy spent by the sensor nodes in ring $\mathrm{i}$ to send those packets and eventually the average energy consumed per node in ring $\mathrm{i}$ :

$$
\left.\left.E_{i}=a p_{i} N_{i} \mid R_{i}^{\alpha}+c\right\rfloor+a\left(r_{i}^{\alpha}+c\right)\left[\left(1-p_{i}\right) N_{i}\right]=a p_{i} N_{i} \mid R_{i}^{\alpha}-r_{i}^{\alpha}\right\rfloor+a N_{i}\left(r_{i}^{\alpha}+c\right)
$$

And average energy consumed per node, $e_{i}=\frac{E_{i}}{m_{i}}=\frac{a p_{i} N_{i}\left[R_{i}^{\alpha}-r^{\alpha}\right]+a N_{i}\left(r_{i}^{\alpha}+c\right)}{\lambda \pi\left(R_{i}^{2}-R_{i-1}^{2}\right)}$

Finally, the hybrid communication algorithm determines the value of probability $\mathrm{p}_{\mathrm{i}},(i=1,2, \ldots, \mathrm{K})$ to ensure uniform energy consumption as follows:

For the last ring:
$p_{k}=\frac{\left(\frac{e_{k}}{a}-\left(r_{k}^{\alpha}+c\right)\right)}{\left(R_{k}^{\alpha}-r_{k}^{\alpha}\right)}$

For the $1^{\text {st }}$ ring:

$p_{i}=1$

For other rings:

$p_{i}=\frac{\frac{e_{i}}{a N_{i}}\left(\lambda \pi R_{i}^{2}-R_{i-1}^{2}\right)-\left(r_{i}^{\alpha}+c\right)}{R_{i}^{\alpha}-r_{i}^{\alpha}}$

\subsection{Proposal 2: Equal Width Hybrid Communication Mode}

The equal width hybrid routing communication mode (referred as hybrid routing) is a combination of the hybrid communication and routing strategy algorithms. The hybrid communication mode will consist of a probability based energy balancing scheme that will divide a circular network into $\mathrm{K}$ rings of equal widths and will find a set of probabilities $P_{i}$ for each ring to balance the energy consumption. A node in ring $i$ will then use the calculated probability $\mathrm{P}_{\mathrm{i}}$ to determine the number of local packets to be forwarded to the next ring $i-1$ towards the $\mathrm{CH}$ and to the $\mathrm{CH}$ directly respectively. Incoming traffic will always be transmitted to the next node in ring $i-1$.

\subsubsection{The equal width hybrid communication algorithm}

The equal width hybrid routing algorithm is similar to the unequal width hybrid algorithm except that the total number of packets in ring $i$ will be as follows:

$$
N_{i}=(2 i-1) \lambda \pi r^{2}+\sum_{j=i+1}^{K}\left(1-p_{j}\right) N_{j}^{L}
$$

\section{IMPLEMENTATION}

The two proposed algorithms have been implemented and compared with the existing equal width hybrid communication scheme (referred as equal width) described in section 2. Below a brief description of the implementation details of the algorithms is given.

\subsection{Implementation details}

In the unequal width hybrid communication algorithm, the widths of the rings differ as the sensors are allowed to adjust their transmission radii. The next step is to determine the number of nodes per ring, their corresponding positions in each ring and deploy them to form a homogeneous cluster, i.e. the sensor nodes are uniformly distributed inside each ring with a known density. Then find the distance between each node and the $\mathrm{CH}$ and consequently the ring number in which the node is located. After determining the optimum value for each ring width, a given node in the $i^{\text {th }}$ ring must now find its neighbouring sensor node which has to be located in the next ring (i.e. $\mathrm{i}-1^{\text {th }}$ ring). The node knows to whom it can be "heard". Each sensor node determines the amount of data to be sent by one-hop direct transmission and by 
multiple hops according to the probability $\mathrm{p}_{\mathrm{i}}$ so as to even out the energy dissipated better.

While determining the probability for each ring to send data packets directly to the $\mathrm{CH}$, the average energy consumed by the sensor nodes in each ring is obtained as well. The algorithm assumes that all sensor nodes located in the same ring are at the same distance from the $\mathrm{CH}$ and each of them has a neighbouring node in the next ring with the same distance between them. But in reality this is not the case as the nodes are homogeneously positioned inside each ring. The calculated transmission probabilities are then used to simulate the proposed algorithms inside the cluster and thereby determine the actual average energy spent per ring.

\section{SIMULATION STUDY}

In this section, the performance of the proposed algorithms is evaluated through simulation where the energy consumption for data transmission from every node to the cluster-head is recorded when varying the radius of the cluster (R), alpha $(\alpha)$ and the number of rings $(\mathrm{K})$. The average energy consumed per ring and transmission probability per ring are observed for the proposed algorithms and the existing equal width hybrid communication scheme. Furthermore comparison between the different algorithms is performed. The simulator that was implemented allows the different parameters to be configured at run time as shown in figure 4 . The simulator also shows the deployment of the nodes and the variation in energy consumption per ring until it is balanced.

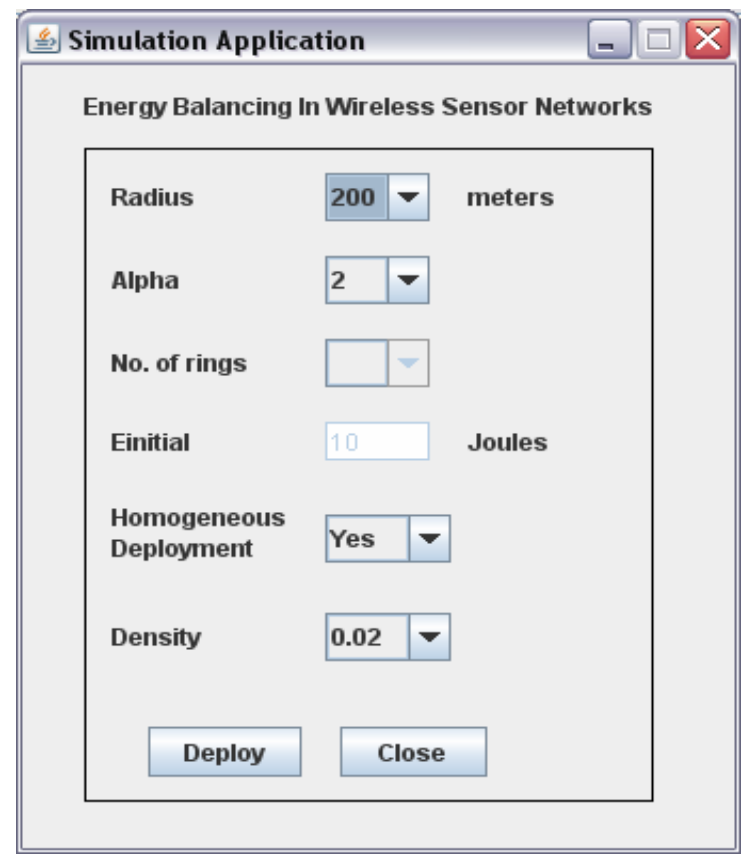

Figure 4: GUI to configure parameters for simulations

Only heterogeneous cluster-based network has been considered in the simulations and each node will generate one packet locally. Furthermore, only homogeneous deployment of the nodes will be considered for the scenarios and the initial energy per node is $10 \mathrm{~J}$. Finally the nodes can communicate directly with the $\mathrm{CH}$ and the density for the different radii of the cluster will be as follows:
$0.006(\mathrm{R}=400 \mathrm{~m}), 0.01(\mathrm{R}=0.03 \mathrm{~m})$ and $0.02(\mathrm{R}=200 \mathrm{~m})$. Using the GUI in figure 4 , the density can be varied only when the $R=200 \mathrm{~m}$ due to memory constraint.

\subsection{Simulation Results}

5.1.1 Scenario 1: Radius of cluster: 200m, Alpha: 3 and Number of rings: 9

Table 1: Average Energy Consumption per ring for scenario 1

\begin{tabular}{|c|c|c|c|}
\hline $\begin{array}{c}\text { Ring } \\
\text { Number }\end{array}$ & $\begin{array}{c}\text { Equal Width } \\
(\mathbf{J})\end{array}$ & $\begin{array}{c}\text { Unequal } \\
\text { Width (J) }\end{array}$ & $\begin{array}{c}\text { Hybrid } \\
\text { Routing (J) }\end{array}$ \\
\hline 1 & 0.006752 & 0.001121 & 0.006753 \\
\hline 2 & 0.004415 & 0.004832 & 0.004519 \\
\hline 3 & 0.003709 & 0.005387 & 0.003837 \\
\hline 4 & 0.003537 & 0.006019 & 0.003609 \\
\hline 5 & 0.003531 & 0.005624 & 0.003717 \\
\hline 6 & 0.003399 & 0.005482 & 0.003551 \\
\hline 7 & 0.003689 & 0.005726 & 0.003872 \\
\hline 8 & 0.003518 & 0.005484 & 0.003616 \\
\hline 9 & 0.003199 & 0.005837 & 0.003341 \\
\hline
\end{tabular}

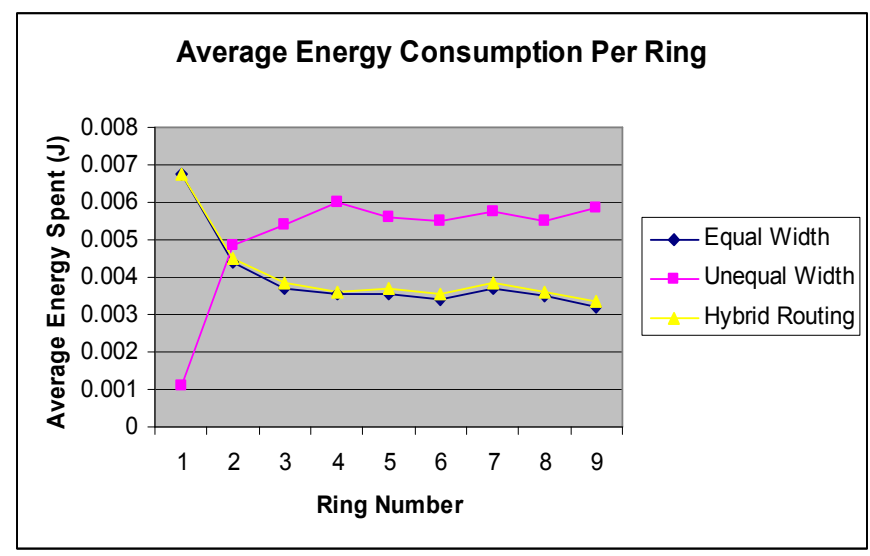

Figure 5: Scenario 1 Average Energy Consumption Rate

Figure 5 shows the average energy spent in the transmission of data packets by the sensor nodes in each ring towards the clusterhead for one round. It can be observed that the variation of energy consumption rate in the equal width and hybrid routing modes are smaller compared to unequal width. However the latter has the lowest maximum energy consumption.

5.1.2 Scenario 2: Radius of cluster: $300 \mathrm{~m}$, Alpha: 3 and Number of rings: 11

Table 2: Average Energy Consumption per ring for scenario 2

\begin{tabular}{|c|c|c|c|}
\hline $\begin{array}{c}\text { Ring } \\
\text { Number }\end{array}$ & $\begin{array}{c}\text { Equal } \\
\text { Width (J) }\end{array}$ & $\begin{array}{c}\text { Unequal } \\
\text { Width (J) }\end{array}$ & $\begin{array}{c}\text { Hybrid } \\
\text { Routing (J) }\end{array}$ \\
\hline 1 & 0.011634 & 0.010126 & 0.015765 \\
\hline
\end{tabular}




\begin{tabular}{|c|c|c|c|}
\hline 2 & 0.012486 & 0.008292 & 0.009539 \\
\hline 3 & 0.013736 & 0.009013 & 0.01005 \\
\hline 4 & 0.012905 & 0.008569 & 0.009082 \\
\hline 5 & 0.012765 & 0.009138 & 0.008428 \\
\hline 6 & 0.012396 & 0.008465 & 0.008751 \\
\hline 7 & 0.012748 & 0.007962 & 0.008309 \\
\hline 8 & 0.012954 & 0.007761 & 0.008716 \\
\hline 9 & 0.012543 & 0.008237 & 0.008528 \\
\hline 10 & 0.01239 & 0.007526 & 0.008528 \\
\hline 11 & 0.013046 & 0.007705 & 0.008812 \\
\hline
\end{tabular}

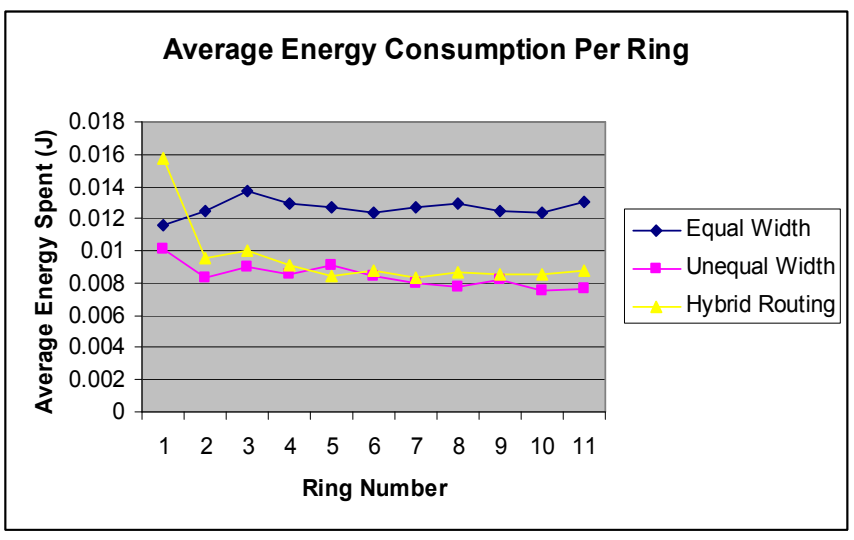

Figure 6: Scenario 2 Average Energy Consumption Rate

Figure 6 shows that the variation in the energy consumption rate in the hybrid routing is higher and it has the maximum energy consumption compared to the others.

\subsubsection{Scenario 3: Radius of cluster: 400m, Alpha: 4 and Number of rings: 15}

Table 3: Average Energy Consumption per ring for scenario 3

\begin{tabular}{|c|c|c|c|}
\hline $\begin{array}{c}\text { Ring } \\
\text { Number }\end{array}$ & $\begin{array}{c}\text { Equal } \\
\text { Width (J) }\end{array}$ & $\begin{array}{c}\text { Unequal } \\
\text { Width (J) }\end{array}$ & $\begin{array}{c}\text { Hybrid } \\
\text { Routing (J) }\end{array}$ \\
\hline 1 & 0.410184 & 0.027297 & 0.481987 \\
\hline 2 & 0.772584 & 0.132929 & 0.489948 \\
\hline 3 & 0.702363 & 0.175868 & 0.405188 \\
\hline 4 & 0.587635 & 0.152822 & 0.374287 \\
\hline 5 & 0.609365 & 0.169613 & 0.382147 \\
\hline 6 & 0.637729 & 0.159044 & 0.414264 \\
\hline 7 & 0.678876 & 0.169099 & 0.418134 \\
\hline 8 & 0.740797 & 0.155483 & 0.454662 \\
\hline 9 & 0.532749 & 0.144243 & 0.242436 \\
\hline 10 & 0.59686 & 0.158609 & 0.41336 \\
\hline 11 & 0.564691 & 0.150376 & 0.210465 \\
\hline 12 & 0.679581 & 0.152459 & 0.352701 \\
\hline 13 & 0.662232 & 0.110101 & 0.340087 \\
\hline
\end{tabular}

\begin{tabular}{|c|c|c|c|}
\hline 14 & 0.419241 & 0.072436 & 0.419241 \\
\hline 15 & 0.49792 & 0.034827 & 0.49792 \\
\hline
\end{tabular}

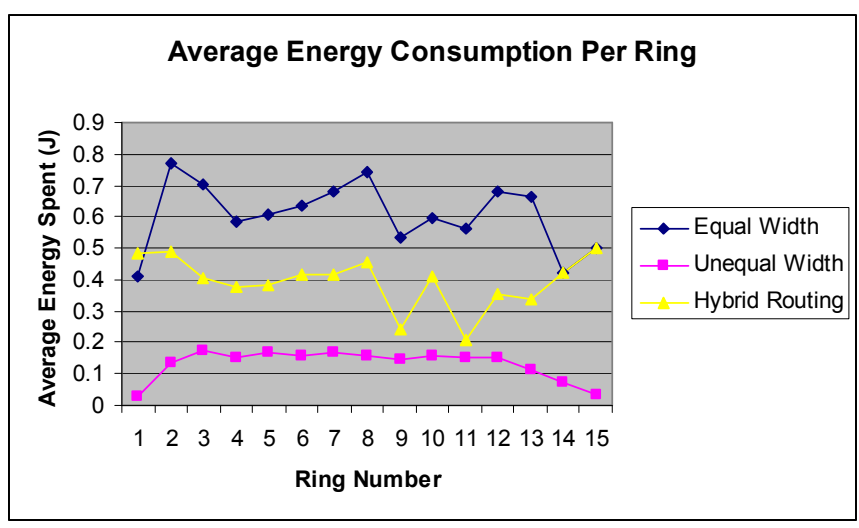

Figure 7: Scenario 3 Average Energy Consumption Rate

Figure 7 shows that the variation of energy consumption in the equal width model is less stable compared to the others. The maximum energy consumption rate is around $0.2 \mathrm{~J}$ for the unequal width, $0.5 \mathrm{~J}$ for hybrid routing while that of equal width is more than $0.7 \mathrm{~J}$. Therefore it can be observed that:

- The average energy consumed is more in the equal width than that in the others. At a point in time, the load on the sensors in one or more rings becomes high and this leads to a high amount of energy consumption (this applies for all algorithms).

- Also, the energy spent is much higher when alpha $=4$ than the one when alpha $=2$ or 3 . This can be explained as follows: enlarging the value of alpha increases the energy consumption used to transmit the same amount of data packets over the same distance.

5.1.4 Scenario 4: Radius of cluster: 400m, Alpha: 2 and Number of rings: 7

Table 4: Average Energy Consumption per ring for scenario 4

\begin{tabular}{|c|c|c|c|}
\hline $\begin{array}{c}\text { Ring } \\
\text { Number }\end{array}$ & $\begin{array}{c}\text { Equal } \\
\text { Width (J) }\end{array}$ & $\begin{array}{c}\text { Unequal } \\
\text { Width (J) }\end{array}$ & $\begin{array}{c}\text { Hybrid } \\
\text { Routing (J) }\end{array}$ \\
\hline 1 & $7.42 \mathrm{E}-04$ & $6.39 \mathrm{E}-04$ & 0.001744 \\
\hline 2 & $6.10 \mathrm{E}-04$ & $6.22 \mathrm{E}-04$ & $6.16 \mathrm{E}-04$ \\
\hline 3 & $6.28 \mathrm{E}-04$ & $6.22 \mathrm{E}-04$ & $5.72 \mathrm{E}-04$ \\
\hline 4 & $6.11 \mathrm{E}-04$ & $6.11 \mathrm{E}-04$ & $5.51 \mathrm{E}-04$ \\
\hline 5 & $6.08 \mathrm{E}-04$ & $6.20 \mathrm{E}-04$ & $5.53 \mathrm{E}-04$ \\
\hline 6 & $6.12 \mathrm{E}-04$ & $6.26 \mathrm{E}-04$ & $5.49 \mathrm{E}-04$ \\
\hline 7 & $6.18 \mathrm{E}-04$ & $6.32 \mathrm{E}-04$ & $5.51 \mathrm{E}-04$ \\
\hline
\end{tabular}

Figure 8: Scenario 4 Average Energy Consumption Rate

\section{INTERPRETATION OF RESULTS}

The network lifetime $\mathrm{L}$ is defined as the number of cycles that the network works until the first sensor node exhausts its energy, that is $L=E_{\text {init }} /\left\{e_{1}, e_{2}, \ldots, e_{\max }\right\} \quad$ where $\mathrm{e}_{\mathrm{i}} \quad(1<=i<=\mathrm{K})$ represents the average energy consumed per ring. 


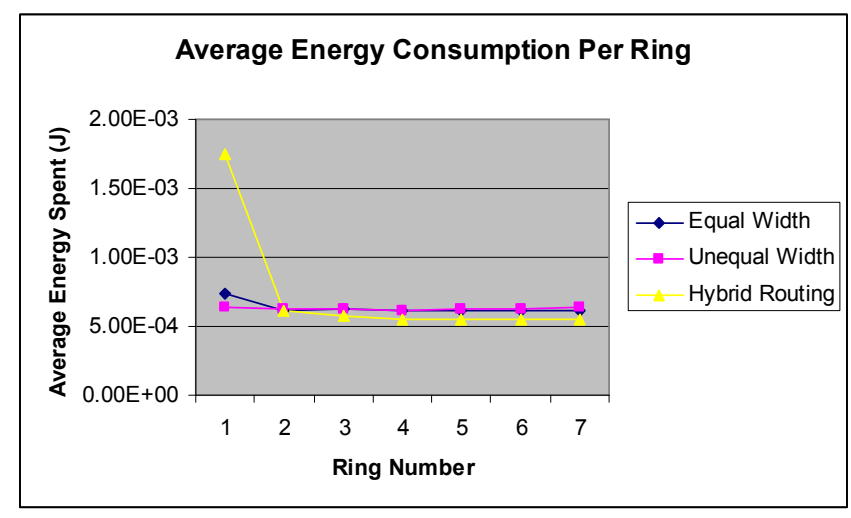

Table 5: Overall Network Life-time

\begin{tabular}{|c|c|c|c|c|c|}
\hline Radius & Alpha & $\begin{array}{c}\text { Number } \\
\text { of rings }\end{array}$ & $\begin{array}{c}\text { Equal } \\
\text { Width }\end{array}$ & $\begin{array}{c}\text { Unequal } \\
\text { Width }\end{array}$ & $\begin{array}{c}\text { Hybrid } \\
\text { routing }\end{array}$ \\
\hline 200 & 2 & 2 & 42373 & 40323 & 42553 \\
\hline 200 & 2 & 3 & 43103 & 42194 & 38610 \\
\hline 400 & 2 & 6 & 14970 & 15198 & 7837 \\
\hline 400 & 2 & 7 & 13477 & 15649 & 5734 \\
\hline 300 & 3 & 11 & 728 & 988 & 655 \\
\hline 300 & 3 & 12 & 635 & 895 & 519 \\
\hline 200 & 4 & 8 & 43 & 123 & 83 \\
\hline 200 & 4 & 11 & 74 & 245 & 123 \\
\hline
\end{tabular}

\section{Observations:}

1. The network lifetime is much longer when alpha $=2$ in comparison when alpha $=3$ or 4 .

2. Overall the unequal width performs as well as both the equal width and hybrid routing techniques but when alpha increases, it works better compared to others.

3. The equal width hybrid routing also balances the overall energy consumption well but however it has been noticed that when $\mathrm{K} \rightarrow \infty$, the total energy consumption reaches $100 \%$ and the gain in lifetime vanishes. Therefore this strategy is useful for smaller $\mathrm{K}$.

4. Unlike hybrid routing, the unequal width hybrid communication mode works well when $\mathrm{K}$ is either large or small.

5. The equal width and hybrid routing communication algorithms are not dependent on the radius of the cluster and work well irrespective of the value of $\mathrm{R}$.

6. From table 22, it can be observed that when the density increases, the average energy consumption per sensor node increases using the unequal width hybrid communication algorithm due to increase in number of nodes while for the hybrid routing technique, it varies due to the load.

7. It has also been noticed that both proposed algorithms work well when the nodes have been deployed randomly and give more or less the same results.

8. Another important point concerning the unequal width algorithm is that it works well even if the unequal width criterion is not satisfied. An example is the first row from table 21 where $R=200 \mathrm{~m}$, alpha $=2$ and $K=2$. Since $r_{1}=$ $67.1 \mathrm{~m}$ and $\mathrm{K}=2$, then by default $\mathrm{r}_{2}=132.9 \mathrm{~m}\left(\mathrm{r}_{2}>\mathrm{r}_{1}\right)$.
9. Finally from the different scenarios, it can be observed that in the equal width hybrid routing algorithm, the sensor nodes closest to the $\mathrm{CH}$ spent higher energy compared to others due to the presence of incoming traffic.

\section{CONCLUSION}

In wireless sensor networks the destination of all the information gathered at the sensor nodes is a single base station and the traffic pattern is highly non-uniform because the nodes, that are closest the base station, have to relay all the data packets. Consequently, those nodes are the first to run out of battery, thereby restricting the lifetime of the network. In this paper the problem of unbalanced energy state in WSN has been considered and two strategies for balancing energy consumption across sensor nodes have been proposed with the aim of prolonging the network lifetime.

In both algorithms a hybrid transmission mechanism, based on probability, is introduced to balance the energy dissipation in the whole network and prolong the network lifetime. In the first algorithm, an unequal-width design is introduced to derive the optimal ring widths which lead to the reduction of energy consumption and prolonging the network lifetime. In the second one, equal ring widths are considered where incoming traffic is always transmitted to the next hop and a probability is used to determine the amount of locally generated packets to be forwarded to the next hop and the cluster-head respectively.

Simulations have shown effectiveness of the proposed techniques which balance the rate of energy consumption across the whole network and extend the overall lifetime of the network efficiently. It has been observed that the unequal width hybrid communication mode is the clear winner in extending the network lifetime as it tries its best to balance as well as minimize the energy consumption of the nodes. The equal width hybrid routing strategy is an alternative to the equal width hybrid communication mode which also works well for energy balancing.

\section{REFERENCES}

[1] Akyildiz, T., Su, W., Sankarasubramanian, Y. and Cayirci, E. 2002. Wireless Sensor Networks: A Survey, Computer Networks, Vol. 38, No. 4, pp. 393-422, March 2002.

[2] Zytoune, O., Fakhri, Y., Aboutajdine, D. 2010. A Novel Energy Aware Clustering Technique for Routing in Wireless Sensor Networks, Journal of Wireless Sensor Network WSN, 2010, 2, 233-238, March 2010.

[3] Lee, D. W., Kim, J. H., and Ko, Y. B. 2005. An Energy Balanced Data Dissemination Scheme for Lifetime Extension in Wireless Sensor Networks", Proceedings of Wireless Networks and Emerging Technologies, Canada, July 2005.

[4] Ai, J., Turgut, D. and Boloni, L. 2005. A Cluster-Based Energy Balancing Scheme in Heterogeneous Wireless Sensor Networks, Proceedings of the 4th International Conference on Networking (ICN'05), Reunion, France, April 2005, pp. 467-474.

[5] Z. Khalid, Z., Ahmed, G., Khan, N. M. and Vigneras, P. 2007. A real-time energy-aware routing strategy for wireless sensor networks, Asia-Pacific Conference on Communications, Bangkok, Thailand, pp. 381-384, 2007. 
[6] Blumenthal, J., Handy, M., Golotowski, F., Haase, M. and Timmermann, D. 2003. Wireless Sensor Networks: New Challenges In Software Engineering, IEEE Conference on Emerging Technologies and Factory Automation, Vol. 1, pp. 551-556, Germany, September 2003.

[7] Haanpaa, H., Schumacher, A., and Orponen, P. 2010. Distributed algorithms for lifetime maximization in sensor networks via Min---Max spanning subgraphs, Wireless Networks, Springer, April 2010, Vol. 16 , No. 3, pp. 875 887.

[8] Le-Huy, P. and Roy S. 2010. Low-Power Wake-Up Radio for Wireless Sensor Networks, Mobile Networks and Applications, Springer, April 2010, Volume 15, Issue 2, pp. $226-236$.

[9] Sun, B., Gao, S., Chi, R. and Huang, F. 2008. Algorithm for balancing energy consumption in wireless sensor networks, Proceedings of the 1st ACM International Workshop on Foundations of Wireless Ad Hoc and Sensor Networking and Computing, China, May 2008.

[10] Wang, X., Ma, J., Wang, S. and Bi, D. 2007. PredictionBased Dynamic Energy Management in Wireless Sensor Networks, Sensors, Vol. 7, pp. 251-266, 2007.
[11] Levendovszky, J., Bojárszky, A. and Oláh, A. 2008. Energy balancing by combinatorial optimization for wireless sensor networks, Wseas Transactions on Communications, Vol. 7, pp. 27-32, 2008

[12] Song, C., Cao, J. N., Liu, M., Zheng, Y., Gong H. G. and Chen, G. 2008. Mitigating Energy Holes Based on Transmission Range Adjustment in Wireless Sensor Networks, Computer Communications, Hong-Kong, July 2008.

[13] Olariu, S. and Stojmenovioc, I. 2006. Design guidelines for maximizing lifetime and avoiding energy holes in sensor networks with uniform distribution and uniform reporting, in Proceedings of IEEE INFOCOM, pp. 1-12, Spain, April 2006.

[14] Liu, Z., Xiu, D. and Guo, W. 2005. An Energy-Balanced Model for Data Transmission in Sensor Networks, in the IEEE $62^{\text {nd }}$ Semiannual Vehicular Technology Conference, Dallas, Texas, September 2005.

[15] Haenggi, M. 2003. Energy Balancing Strategies For Wireless Sensor Networks, In the Proceedings of the 2003 International Symposium on Circuits and Systems, Vol. 4, pp. 828-831, USA, 2003 